\title{
Blind Detection Method for Video Inpainting Forgery
}

\author{
Sreelekshmi Das Gopu Darsan Shreyas L Divya Devan \\ Department of Computer Science and Engineering \\ Kerala University \\ Sree Buddha College of Engineeing \\ Kerala, India
}

\begin{abstract}
Video forgery, also referred as video falsifying, is a technique for generating fake videos by altering, combining or creating new video contents. Exemplar-based inpainting technique can be used to remove objects from an image/video and play visual tricks, which would affect the authenticity of videos. In this paper, a blind detection method based on zero-connectivity feature and fuzzy membership function is proposed to detect the video forgery. Firstly, the forged video is converted into frames, then zero-connectivity labelling is applied on block pairs to yield matching degree feature for all blocks in the forged region and construct ascending semi-trapezoid membership for computing fuzzy membership function. Finally, the tampered regions are identified using a cut set.
\end{abstract}

\section{Keywords}

Video forgery; Exemplar-based inpainting; Zero-connectivity labelling; Fuzzy Membership; cut set

\section{INTRODUCTION}

Inpainting is the process of reconstructing lost or deteriorated parts of images and videos. For instance, in the case of a valuable painting, this task would be carried out by a skilled image restoration artist. Inpainting is also used for removing objects from digital images/videos and fill that area with matching background content. Figure 1 shows an example of this task, where the foreground person is manually selected as the target region and replaced by textures sampled from the remainder of the image.

Although the research of altering contents in a video may create a sociological problem, it is interesting and challenging one to investigate video forgery, also referred as video falsifying, technologies if they are used with good intension e.g. Special effects in a movie. Digital image/video tampering has become major problem lately, due to ease of artificially synthesizing photographic or video fake -for promoting a story by media channels and social networking websites. This is due to significant advances in computer graphics and animation technologies, and availability of digital video/image manipulation and cloning tools. With lack of proper regulatory frameworks and infrastructure of prosecution of such evolving cyber-crimes, there is an increasing dissatisfaction about increasing use of such tools for law enforcement, and a feeling of cynicism and mistrust amoung the civilian operating environments. The availability of digital image processing tools, such as photoshop, makes it relatively easy to remove or add important features from an image/video without leaving any proof of tampering

Digital video/image forgery detection can use either active forgery detection techniques or passive forgery detection techniques. A significant body of work, however is available on active forgery detection techniques, which involves embedding digital watermark into the images when the images are captured. The problem with active forgery detection techniques is that not all camera manufacturers embed the watermarks, and in general, most of the customers have dislike towards camera which embed watermarks due to compromise in the image quality. So there need a passive and blind forgery detection techniques with no watermark embedded in the images.
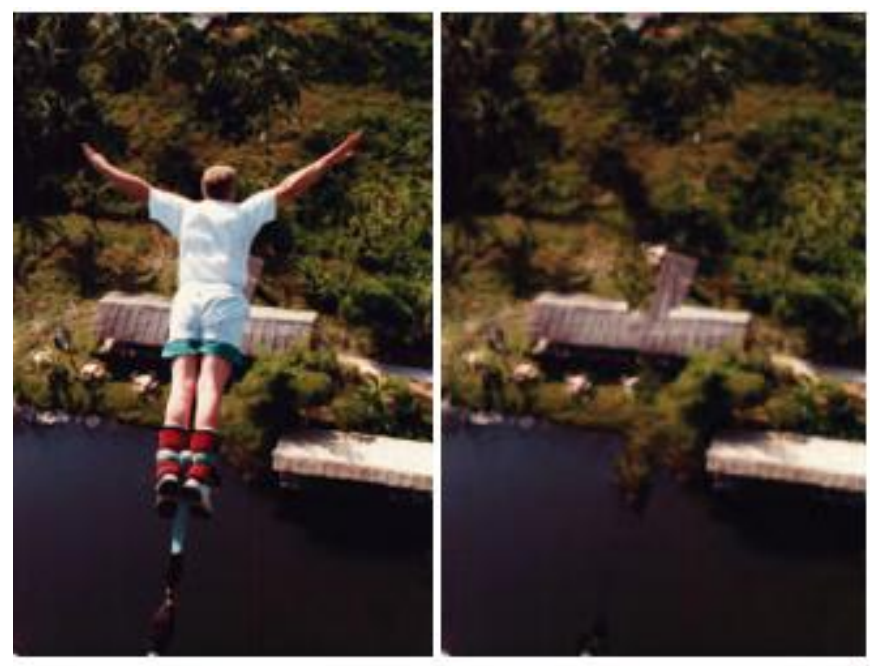

(a)

(b)

Fig1: (a) orginal image (b) The region corresponding to the foreground person has been manually selected and then automatically removed.(Courtesy: Google-Object removal by exemplar based image inpainting.)

For detecting video forgery, falsified video is converted to several frames and the blind detection method based on zeroconnectivity feature and fuzzy membership is proposed to detect the forgery. Since digital image forgery is a challenging problem and lack need to be continuously enriched and improved.

In contrast to above approaches, we describe a method for detecting tampered video/image created by inpainting technique. Inpainting is a method to fill the missing area in a image using the information from the remaining portion of the image. Region filling and object removal using inpainting technique is important both for content correction by removing unwanted objects and for image restoration by repairing 
damaged area. But it is also a key tool for manipulation of image, such as producing photo-montages, so it is necessary to develop a forensic algorithm to detect the inpainted tampered video/images. Although this inpainted doctoring is often imperceptible, it introduces abnormal similarity into the image block pairs, which can be used as evidence for digital forgery. In this paper we describe the form of this abnormal similarity, and how they can be automatically detected in any region of an image/video.

\section{RELATED WORK}

Recently, several image forensics methods for detecting digital forgeries were proposed, in the complete absence of any form of digital signature or watermark. Firstly, Fridrich established methods for detecting copy-move forgeries [1] and double compressed JPEG images [2]. After that Johnson proposed several methods for exposing digital forgeries through inconsistencies in lighting [3] and specular highlights on the eye [4]. Popescu introduced method for exposing digital tampering in color filter array interpolated images [5]. In [6][7], several statistical approaches are proposed to detect image splicing. In [8], Bayram proposed binary similarity measure(BSM), image quality measure(IQM) and high order wavelet(HOW) statistics features are extracted to detect image manipulation. In [9] Dan tu established a blind detection method for exemplar based inpainting forgery. Mehdi Ghorbani introduced DCT-DWT based image forgery detection technique[11]. Asok De proposed detection of forgery in digital video[12].

The proposed method in this paper preserves dividing falsified video into several frames and the blind detection method based on zero-connectivity feature and fuzzy membership is applied to detect the forgery.

\section{EXEMPLAR-BASED INPAINTING}

In this section, we review exemplar-based inpainting algorithm proposed by Criminisi [14] which is for removing large objects and filling in the hole with similar background content.

Criminisi's algorithm iterates the following three steps until all pixels have been filled: (i) Computing patch priorities. Patch priorities determine the order of repair, and repair order has important implications to the ultimate outcome.(ii) Propagating texture and structure information. According to texture similarity, the algorithm searches best-match patch in the source region for to be filled patch.(3) Filling to be filled patch. The value of each pixel to be filled is copied from its corresponding position inside best match patch. Subsequently there have been some improvements on Criminisi's algorithm, which mostly concentrate on improving priority calculation and optimizing patch searching method.

Despite there are differences in ways of calculating priority and searching among Criminisi's algorithm and its improved algorithms, the final step of copying best match block and replacing the block to be filled will introduce abnormal similarity between the repaired region and the rest region of the image. This abnormal similarity can be used as a basis for successful detection of inpainted forged images/video.

\section{PROPOSED DETECTION METHOD}

For detecting video forgery, falsified video is converted into several frames (shown in figure 2) and the blind detection method based on zero-connectivity feature and fuzzy membership is proposed to detect the forgery.

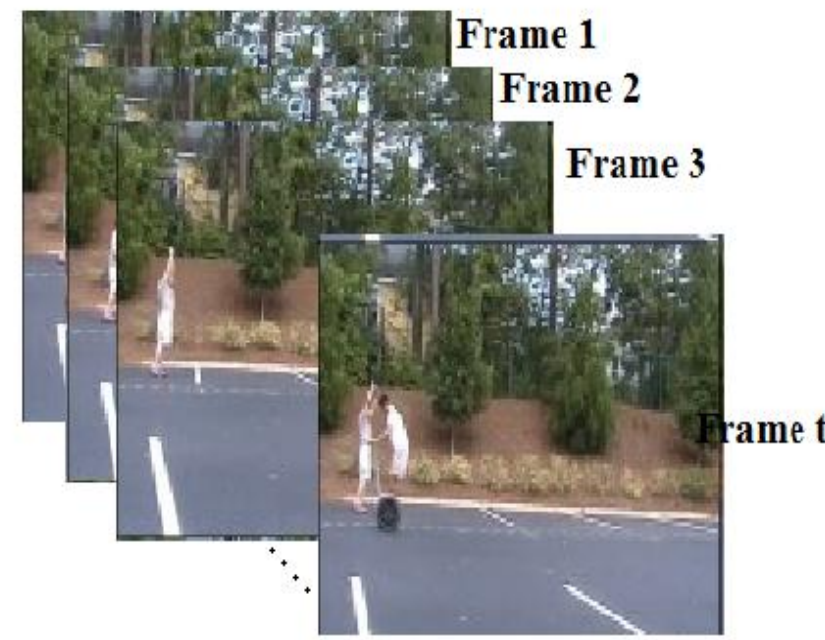

Fig 2: A video is divided into number of frames.

For ease of understanding, we adopt the notation similar to used in the Criminisi's literature. First, given an input forged video, the user convert the video into frames and select the region of suspicious (ROS), $\Omega$, which may be defined as the entire image or part of it. The purpose of selecting partial region as ROS is to produce good balance between complexity and performance. Then the size of template, $\mathrm{m} * \mathrm{n}$, the parameters of the membership function a,b, and the threshold of cut set $\lambda$ should be specified. Once these parameters are defined, our algorithm iterates the following three steps until all blocks in the ROS have been checked. these parameters are defined, our algorithm iterates the following three steps until all blocks in the ROS have been checked.

In this paper we describe steps for video forgery detection. The steps could be summarised as firstly the given forged video is converted into number of frames and the apply the blind detection method based on zero-connectivity feature and fuzzy membership function to detect the forgery.

\subsection{Computing Block Matching Degrees}

Due to the filling scheme of exemplar-based inpainting algorithm proposed by Criminisi, there exist a number of zeros connecting in the difference array of filling block and its best match block. Based on this characteristic, we adopt the length of largest zero connectivity set in the block pair difference array as the corresponding block matching degree. Zeroconnectivity should meet following conditions simultaneously: (i) the difference of corresponding pixel in the block pair is zero. (ii) ther still exists zero or zeros within its 8neighbourhoods, that is, the location and other zero positions are 8-neighbourhood connected.

For each block $\Psi p$ of the ROS where $\mathrm{p}$ is its upper left point, we search in the rest region of the image $\Phi=I-\Psi p$ for that block, which is the best similar to $\Psi p$. Formally

$$
\Psi \hat{q}=\arg \max _{\Psi q \in \Phi} n(\Psi p, \Psi q)
$$

Where $n(\Psi p, \Psi q)$ is the matching degree of block pair $(\Psi p, \Psi q)$.After having found the best-similar block $\Psi \hat{q}$, the matching degree of $\Psi p$ is the matching degree of block pair $(\Psi p, \Psi \hat{q})$ : 


$$
\sigma \Psi p=n(\Psi p, \Psi q)
$$

The matching degree $n(\Psi p, \Psi q)$ is computed as: 1$)$ two blocks are subtracted, then fake their absolute values and obtain difference array $D p q$, see equation(3); 2) in order to extract the number and location relations of "zero" appeared in Dab, it is necessary to label all zero components. We use binary image component labelling method to extract zero connectivity features. Connectivity determines whether two binary pixels are connected or not. 4-connectivity and 8-connectivity are commonly used, and the pixels are marked by coordinates that are considered connected to $\mathrm{P}$. A pixel, $\mathrm{Q}$, is a 4-neighbour of a given pixel, $\mathrm{P}$, if $\mathrm{P}$ and $\mathrm{Q}$ share an edge. The 4-neighbour of pixel P (namely pixels P2,P4,P6 and P8) are shown in Figure 4 below. First $D p q$ should be converted into binary array by applying (4), where t denotes the threshold. Setting a suitable t can be remove noise impact noise impact while maintaining good performance. In this paper we define $\mathrm{t}=0$. Having found all the connectivity components sets in, each set is marked by a unique label. Suppose the largest label is marked by "N", then only the greatest length among all labels is taken as its matching degree $n(\Psi p, \Psi q)$.

$$
D p q=a b s(\Psi p-\Psi q)
$$

$$
B W p q(i, j)=\left\{\begin{array}{lc}
1 & D p q(i, j) \leq t \\
0 & D p q(i, j)>t
\end{array}\right.
$$
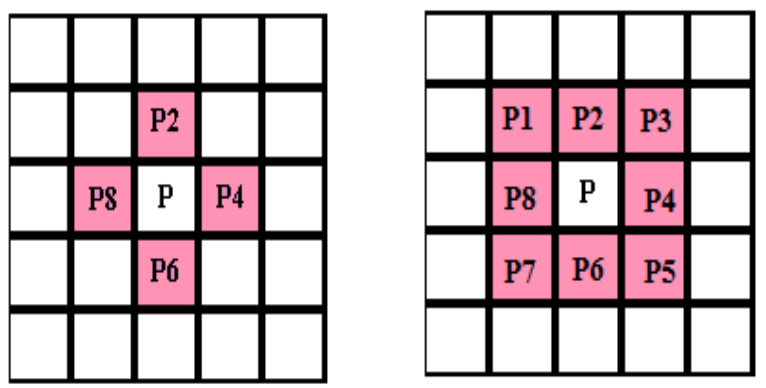

Fig 3: 4-connectivity and 8-connectivity diagram

\subsection{Computing Fuzzy Membership}

Yet another problem in our method is how to convert the characteristics of block matching degrees into the judging factors of image doctoring. No matter how accurate our measurements (e.g. block matching degree) are some uncertainty always remains, therefore we use fuzzy set theory to describe the uncertainty in detecting tampered regions. Fuzzy set theory is the extension of conventional set theory. It handles the concept of partial truth. It was introduced by Prof. Zadeh in 1965 as a mean to model the vagueness and uncertainty in complex systems . A fuzzy set is characterized by a membership function, which maps the members of the universe into the unit interval[ $[0,1]$.
Let the universe set $\mathrm{U}$ denotes block matching degree, the fuzzy set $\tilde{\mathrm{A}}$ denotes "tampered block", then $\tilde{\mathrm{A}}$ is defined by its membership function:

$$
\mu_{\tilde{A}}: U \rightarrow[0,1]
$$

A membership function $\mu \tilde{\mathrm{A}}$ is defined as:

$$
\mu_{\tilde{A}}(\sigma \Psi p ; a, b)=\left\{\begin{array}{l}
0 \quad \sigma_{\Psi_{\mathrm{p}}} \leq a \\
\frac{\sigma_{\Psi_{p}}-a}{b-a} \quad a<\sigma_{\Psi_{p}} \leq b \\
1 \quad \sigma_{\Psi_{\mathrm{p}}}>\mathrm{b}
\end{array}\right.
$$

(7)

Because the fuzzy set described here is preferred to big fuzzy phenomenon, therefore we select an ascending semitrapezoidal membership function.

\subsection{Dividing the Cut Set}

Fuzzy set is defined by the membership function, and we agree that if the membership $\mu_{\tilde{A}}(\sigma \Psi p)$ meets or exceeds the threshold $\lambda$, then $\sigma \Psi p$ belongs to the cut set. A cut set $A_{\lambda}$ of a fuzzy set $\tilde{A}$ is a crisp set which defined by:

$$
A_{\lambda}=\left\{\sigma \Psi p \mid \mu_{\tilde{A}}(\sigma \Psi p) \geq \lambda, \sigma \Psi p \in U\right\}, 0 \leq \lambda \leq 1
$$

Finally, if $A_{\lambda} \neq \Phi$, for all matching degrees in $A_{\lambda}$, the corresponding blocks that contributed to that specific matching degree are colored with the white color and thus identified as regions that might have been forged. In addition, another assumption that should be accepted is that forged region will be likely be a connected component rather than a collection of very small blocks or individual pixels. Therefore in this method, morphological operations are employed for removing isolated blocks which may be introduced by noise or compression [17].Morphological operations used here include dilation and erosion, and they can be defined as:

$$
\begin{aligned}
& f \oplus B=\{X \mid B(X) \cap X \neq \Phi\} \\
& f \Theta B=\{X \mid B(X) \subseteq f\}
\end{aligned}
$$

Where $\mathrm{f}$ denotes 2-dimension image, $\mathrm{B}$ denotes structuring element, x denotes pixel value, $\oplus$ denotes dilation operation, and $\Theta$ denotes erosion operation.

The block diagram of proposed system is shown in Figure 4. In block diagram, firstly the given forged video is converted into number of frames and then the blind detection method which is based on zero-connectivity feature is applied to it .Later in this fuzzy membership function is calculated. This gives a cut set value is used to decide the forgery in video frames as it characterises the forged and unforged parts in the video . 


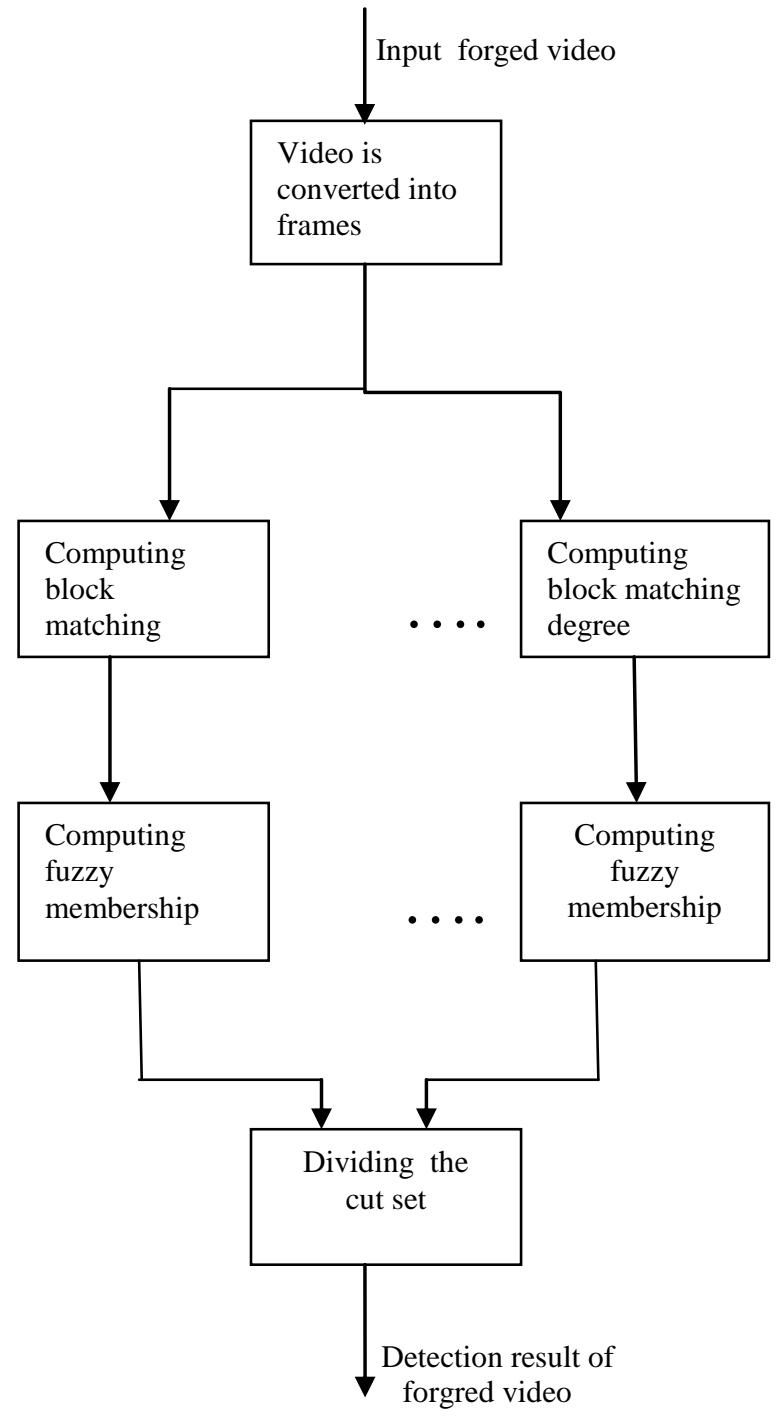

Fig 4: Block diagram of proposed system

A pseudocode description of the algorithmic steps is shown in below.

- Given an input image I.

- Select ROS $\Omega$, set parameters $\Psi, \mathrm{a}, \mathrm{b}, \lambda$

- For $\forall p \in \Omega$, repeat the following steps:

1a.Compute zero-connectivity length $n(\Psi p, \Psi q)$,

$\forall \Psi q \in \Phi=I-\Psi p$

1b.Find the patch $\Psi \hat{q}$ with the maximum zero-

connectivity length,i.e. $\Psi \hat{q}=\arg \max n(\Psi p, \Psi q)$ $\Psi q \in \Phi$

1c. Compute Match Degree $\sigma \Psi p=n(\Psi p, \Psi q)$

2.Compute fuzzy membership $\mu_{\tilde{A}}(\sigma{ })$.

3.Extract cut-set $A_{\lambda}$

- If $A_{\lambda} \neq \Phi$, locate tampered region; else exit.
We can compare our proposed method to those of existing algorithms as shown in Table 1 .

Table 1: Comparison with existing algorithms.

\begin{tabular}{|l|l|l|l|}
\hline Algorithm & $\begin{array}{l}\text { Applicable } \\
\text { for }\end{array}$ & $\begin{array}{l}\text { Method } \\
\text { used }\end{array}$ & Performance \\
\hline Tzu & $\begin{array}{l}\text { Video } \\
\text { inpainting } \\
\text { forgery }\end{array}$ & $\begin{array}{l}\text { Correlation } \\
\text { of noise } \\
\text { residue }\end{array}$ & $\begin{array}{l}\text { Fast but need } \\
\text { more accurate. }\end{array}$ \\
\hline Mehdi & $\begin{array}{l}\text { Image } \\
\text { inpainting } \\
\text { forgery }\end{array}$ & DWT-DCT & $\begin{array}{l}\text { Simple one but } \\
\text { not suitable for } \\
\text { all images. }\end{array}$ \\
\hline Zhang & $\begin{array}{l}\text { Image } \\
\text { inpainting } \\
\text { forgery }\end{array}$ & SVD & $\begin{array}{l}\text { Need more } \\
\text { accurate. }\end{array}$ \\
\hline Proposed & $\begin{array}{l}\text { Both video } \\
\text { and image } \\
\text { inpainting } \\
\text { forgery }\end{array}$ & $\begin{array}{l}\text { Blind } \\
\text { detection } \\
\text { method }\end{array}$ & $\begin{array}{l}\text { Fast and more } \\
\text { accurate } \\
\text { result. }\end{array}$ \\
\hline
\end{tabular}

Here we can apply our method to a variety of inpainted forged videos and images. In all experiments, consider each frames and parameters are set to: $m * n=9 * 9, a=4, b=78, \lambda=0.5$.

We can apply our method to a variety of inpainted forged images and video. Figure 5(a)(d) are original natural video; Figure 5(b)(e) is inpainted video; Figure 5(c)(f) is expected detection result using our proposed method, here the forged region is colored with white color.

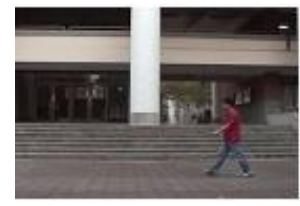

(b)

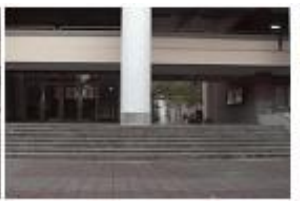

(c)

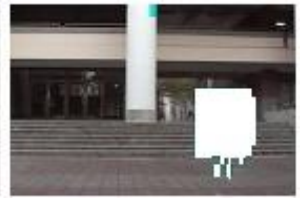

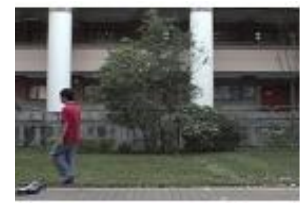

(d)

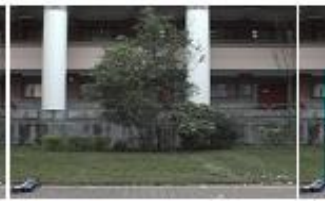

(e)
Fig 5:(a)(d) Source video frame. (b)(e) Correspondingfalsified result of (a) .(c)(f)Detection result of an inpainted forged video.

\section{CONCLUSION}

For detecting video forgery, falsified video is converted into several frames and the blind detection method based on zeroconnectivity feature and fuzzy membership is proposed to detect the video forgery. The exemplar based inpainting process typically leaves behind no perceptual artifacts, it does introduce abnormal similarity between image block pairs. We have presented an efficient technique that automatically detects forged regions in an inpainted video/image. The technique works by applying zero connectivity labelling on block pairs to yield matching degree feature of all blocks in ROS. Fuzzy memberships are then computed by constructing ascending semi-trapezoidal membership function, and tampered regions are identified by a cut set. The major weakness of our approach is that it is currently only applicable to uncompressed images and video. 
Our future work will proceed to detect forged video/image created by other inpainting techniques, so that blind video forensics can be used in various forgery circumstances.

\section{ACKNOWLEDGMENT}

Our thanks to all the faculty members and colleagues who have contributed towards development of this paper.

\section{REFERENCES}

[1] J. Fridrich, D. Soukal, J. Lukáš, "Detection of copymove forgery in digital images," In: Proc. Digital Forensic Research Workshop, Cleveland, OH, 2003.

[2] J. Lukáš, J. Fridrich, "Estimation of primary quantization matrix in double compressed JPEG images," In: Proc. of DFRWS, Cleveland, OH, USA, 2003.

[3] M. Johnson, H. Farid, "Exposing digital forgeries by detecting inconsistencies in lighting," In: Proc. of ACM Multimedia and Security Workshop, New York, NY, 2005.

[4] M. Johnson, H. Farid, "Exposing digital forgeries through specular highlights on the eye," In: Proc. of 9th International Workshop on Information Hiding, Saint Malo, France, 2007.

[5] A. Popescu, H. Farid, "Exposing digital forgeries in color filter array interpolated images," IEEE Transactions on Signal Processing, 2005, 53(10): 3948-3959.

[6] T.-T. Ng, S.-F. Chang, "A model for image splicing," In: IEEE International Conference on Image Processing, Singapore, 2004.

[7] Y. Q. Shi, C. H. Chen, "A natural image model approach to splicing detection," In: Proc. of MM\&Sec, 2007, 5162.

[8] S. Bayram, I. Avcibas, B. Sankur, "Image manipulation detection," Journal of Electronic Imaging, 2006, 15(4). of Noise Residue" 2008.

[9] Qiong WU, Shao-Jie Sun, Wei Zhu, Guo-Hui Li, Dan Tu "Detection of digital doctoring in exemplar-based inpainted images", 2008
[10] Chih-Chung Hsu "Video Forgery Detection Using Correlation of Noise Residue" 2008.

[11] Mehdi Ghorban "DCT-DWT based image forgery detection technique" 2009.

[12] Asok De, Sparsh Gupta, Himanshu Chadha "Detection of forgery in digital video", 2009.

[13] Patrick P'erez, Michel Gangnet, Andrew Blake, "Patchworks: example-based region tiling for image editing," Microsoft Research Technical Report, MSR-TR2004-04.

[14] A. Criminisi, P. Pérez, and K. Toyama, "Object removal by exemplar based inpainting," In: Proc. Conf. Computer Vision and Pattern Recognition, Madison, WI, June 2003.

[15] Wei Zhu, Guo-Hui Li, Dan Tu, "Application of texture synthesis in old photograph completion," Computer Engineering and Application, 2007, 28(10): 220-222.

[16] F. Tang, Y. T. Ying, et al, "A novel texture synthesis based algorithm for object removal in photographs," In: Proc. of 9th Asian Computing Science Conference, Thailang, 2004, 248-258.

[17] R. C. Gonzalez, R. E. Woods, S. L. Eddins, Digital Image Processing Using Matlab. Prentice Hall, 1st edition, 2003.

[18] G. J. Klir, U. St. Clair, B. Yuan, Fuzzy Set Theory:Foundations and Applications, Prentice Hall, 1997.

[15] F. Nielsen and R. Nock, "ClickRemoval: Interactive pinpoint image objectremoval," in Proc. 13th Annu. ACM Int. Conf. Multimedia 2005, pp. 315-318.

[16] K. A. Patwardhan, G. Sapiro, and M. Bertalmio, "Video inpainting of occluding and occluded objects," in Proc. 2005 IEEE Int. Conf. ImageProcess., Genova, pp. II, pp. $69-72$ 